\title{
Hypercholesterolemia and COVID-19: Statins for Lowering the Risk of Venous Thromboembolism
}

\author{
Alpo Vuorio ${ }^{1,2 \star}$, Riitta Lassila ${ }^{3}$ and Petri T. Kovanen ${ }^{4}$ \\ ${ }^{1}$ Department of Forensic Medicine, University of Helsinki, Helsinki, Finland, ${ }^{2}$ Mehiläinen Airport Health Centre, Vantaa, \\ Finland, ${ }^{3}$ Research Program Unit in Systems Oncology, Coagulation Disorders Unit, Helsinki University Hospital, University of \\ Helsinki, Helsinki, Finland, ${ }^{4}$ Wihuri Research Institute, Biomedicum Helsinki 1, Helsinki, Finland
}

Keywords: COVID-19, statins, venous thromboembolism, familial hypercholesterolemia, LDL cholesterol

OPEN ACCESS

Edited by:

Ricardo Gómez-Huelgas,

Regional University Hospital of

Malaga, Spain

Reviewed by:

Joseph Aslan

Oregon Health and Science University,

United States

Ibra S. Fancher,

University of Delaware, United States

Changcheng Zhou,

University of California, Riverside,

United States

*Correspondence:

Alpo Vuorio

alpo.vuorio@gmail.com

Specialty section:

This article was submitted to

Lipids in Cardiovascular Disease,

a section of the journal

Frontiers in Cardiovascular Medicine

Received: 19 May 2021

Accepted: 09 September 2021

Published: 13 October 2021

Citation:

Vuorio A, Lassila $R$ and Kovanen PT (2021) Hypercholesterolemia and COVID-19: Statins for Lowering the Risk of Venous Thromboembolism. Front. Cardiovasc. Med. 8:711923.

doi: 10.3389/fcvm.2021.711923

\section{INTRODUCTION}

In this Opinion letter, we focus on the prevention of venous thromboembolism in hypercholesterolemic patients suffering from COVID-19. We present the heterozygous form of familial hypercholesterolemia $(\mathrm{HeFH})$ as the prototype of genetically determined pure hypercholesterolemia. Moreover, we examine the special therapeutic challenges that one faces when treating a COVID-19 patient suffering from the common (non-HeFH) form of hypercholesterolemia.

$\mathrm{HeFH}$ is an autosomal dominant disorder resulting in a lifelong two- to three-fold elevation of serum low-density lipoprotein cholesterol (LDL-C) $(1,2)$. According to a recent report including 62 studies and over 7 million individuals, the overall prevalence of $\mathrm{HeFH}$ is about 1:300 among the general population worldwide $(3,4)$. Thus, roughly 30 million people suffer from HeFH. Most importantly, severe hypercholesterolemia leads to about 10 -fold increased risk for premature atherosclerotic cardiovascular disease (ASCVD), premature atherothrombotic complications being the leading cause of mortality in this disease (5). In addition to the highly elevated serum LDL-C, patients with $\mathrm{HeFH}$ tend to have serum lipoprotein(a) $[\mathrm{Lp}(\mathrm{a})]$ levels that are higher than in the general population (6).

The Lp(a) particles are LDL-like lipoprotein particles in that they carry cholesterol which contributes to cholesterol accumulation in the developing atherosclerotic lesions. Moreover, the $\mathrm{Lp}$ (a) particles carry proinflammatory oxidized phospholipids and thus contributing also to the inflammatory reactions of the atherosclerotic arterial wall, including promotion of endothelial dysfunction (7). Since the plasma level of $L p(a)$ particles is genetically determined, an increased level constitutes a life-long atheroinflammatory burden to the arterial wall. Finally, the $\mathrm{Lp}(\mathrm{a})$ particles are prothrombotic since they activate endothelial cells and platelets and impair spontaneous fibrinolysis of a developing thrombus $(6,8,9)$.

Continuous and effective blood cholesterol-lowering therapy increases the life expectancy of the $\mathrm{HeFH}$ patients approximately to the level of the general population (10). Effective LDL-C-lowering with a statin should be started in children 8-10 years old (11). Moreover, multidrug pharmacotherapy in which ezetimibe or a PCSK9 inhibitor is added to the statin monotherapy is deemed safe and effective. When needed to reach an effective LDL-C lowering, such combination therapy could be initiated already in adolescent HeFH patients (11-14). 


\section{COVID-19 AND VENOUS THROMBOEMBOLISM}

In a recent meta-analysis involving hospitalized patients with COVID-19, the incidence of deep vein thrombosis (DVT) was $11.8 \%(7.1-17.4)(95 \%, \mathrm{CI})$, and that of pulmonary embolism (PE) was $13.5 \%(8.4-29.5)(95 \%, C I)(15)$. In a later French nationwide retrospective cohort study, which included 89530 hospitalized COVID-19 patients, the incidence of VTE (without or with $\mathrm{PE}$ ) was $4.9 \%$ and that of $\mathrm{PE} 3.4 \%$, these incidences being much higher than those for patients hospitalized, e.g., for seasonal influenza (16).

Patients with CHD carry, in general, a higher risk for VTE than patients without CHD (17). However, among critically ill COVID-19 patients, only limited data are available on the coexisting risk of VTE and CHD. Thus, in a study of critically ill patients with COVID-19 and having thromboprophylaxis, only 2 of 88 patients had $\mathrm{CHD}$, and both belonged to the group which had developed VTE $(N=40)$ (18). Of note, overweight, hypertension and diabetes are additional risk factors for VTE in patients with COVID-19. Furthermore, the incidence of COVID-19-associated arterial thrombosis involving the coronary, cerebrovascular, and peripheral arteries was reported to be around $4 \%$ (19). Although we have learned to improve and intensify the thromboprophylactic strategies after the first waves of the pandemic, the above studies show that despite such efforts, a risk for thrombotic and thromboembolic complications still prevails.

\section{STATINS AND COVID-19}

Ever-increasing numbers of evidence-based data derived from individual studies and meta-analyses show that statins are beneficial for the prognosis of COVID-19 patients and, accordingly, a consensus expert statement calls for a continuation of statin treatment in COVID-19 patients also after the illness (20-24). The amenable effect of statins especially applies to patients with $\mathrm{HeFH}$ because of both the magnitude and duration of the elevation of serum LDL-C, which often combines with an elevated $\mathrm{Lp}(\mathrm{a})$ level and then jointly correlates with the severity of systemic endothelial dysfunction and coagulation propensity (25). Of note, however, statins fail to lower Lp(a) levels.

Statins reduce thrombin and fibrin formation (26-28). Indeed, in many epidemiological studies, this class of drugs has been shown to prevent thrombosis. The preventive action of statins also applies to the COVID-19-affected venous system and the development of $\mathrm{PE}$, which themselves pose additional significant threats in severe COVID-19 on top of the microvascular damaging effect of the SARS-CoV-2 infection (29). In addition, the anti-inflammatory effects of statins have been shown in a cytokine interaction model involving cocultured human muscle and mononuclear cells (30). Especially among the high-risk $\mathrm{HeFH}$ patients, statin-induced improvement of endothelial function may decrease the incidence of both arterial thrombosis, typically manifested as acute myocardial infarction (AMI), and $\operatorname{VTE}(31,32)$.

\section{FAMILIAL HYPERCHOLESTEROLEMIA AND VENOUS THROMBOEMBOLISM}

Since $\mathrm{HeFH}$ (like in other hypercholesterolemias), LDL-C accumulates in the thick subendothelial inner layers of the arterial walls but not in the thin subendothelial layers of veins, it is evitable that the atherosclerotic changes in the arterial system determine the clinical outcome in this disease. However, since high levels of LDL-C and $\mathrm{Lp}$ (a) cause endothelial dysfunction throughout the vascular system, including the venous endothelium, thrombotic and thromboembolic clinical events may also ensue. Yet, data on whether the $\mathrm{HeFH}$ patients, in general, have an increased risk for VTE are minimal, one likely reason being the fact that, in general, $\mathrm{HeFH}$ remains heavily underdiagnosed throughout the world (33). This apparently puzzling issue remains a challenge for future studies because in the general population hypercholesterolemia is a risk factor for VTE (34), and statins show primary prevention effects on VTE (35). In the study by Kawasaki et al. (34), including 109 patients with DVT and 109 controls, even modest elevations of serum total cholesterol level (equal to or above $220 \mathrm{mg} / \mathrm{dl}$ or 5.7 $\mathrm{mmol} / \mathrm{l}$ ) did associate with an elevated risk of DVT (OR 2.6). Therefore, we may surmise that in untreated $\mathrm{HeFH}$ patients, the exceptionally high cholesterol levels will damage the venous endothelium.

Krogh et al. (36) reported that in $\mathrm{HeFH}$, the prevalence of VTE was $\sim 14 \%$ among 60-year-old male and female patients, and according to calculations the annual incidence rate is $2 / 1,000$. Based on the systematic review of VTE, the incidence of VTE among the 60 -year-old $\mathrm{HeFH}$ patients is close to that in 10-20 years older persons in the general population [26]. Accordingly, the severe hypercholesterolemia observed in untreated or undertreated $\mathrm{HeFH}$ patients, i.e., in the majority of $\mathrm{HeFH}$ patients, increases the risk of VTE. Given that globally the majority of $\mathrm{HeFH}$ patients remains undiagnosed, and even if diagnosed, they remain undertreated (33), we can infer that the vast majority-if not all-of the HeFH patients with COVID-19 carry an elevated risk of VTE. Provided the hypercholesterolemia of an $\mathrm{HeFH}$ patient is accompanied by other systemic endothelial-damaging risk factors, such as hypertension, hyperglycemia, or obesity, the risk of VTE must be still increased.

We have recently pointed to the potentially beneficial effects of cholesterol-lowering therapy in preventing endothelial dysfunction and thrombotic events in $\mathrm{HeFH}$ patients with COVID-19, particularly when a statin is combined with a PCSK9 inhibitor (37). Regarding the thrombotic and thromboembolic risk, it is important to note that the cholesterol-lowering drugs also decrease the turnover of fibrin, i.e., the statins decrease Ddimer levels by about 15\%, and the PCSK9 inhibitors decrease the plasma level of the anti-fibrinolytic Lp(a) particles by about $30 \%(38,39)$. The Lp(a)-lowering effect of a PCSK9 inhibitor appears to prevent recurring acute coronary syndrome (ACS) in patients on maximally tolerated statin therapy which has 
already resulted in a low LDL-C level (around $70 \mathrm{mg} / \mathrm{dl}$ or 1.8 $\mathrm{mmol} / \mathrm{l})(40)$. Therefore, we advocate using a PCSK9 inhibitor in hospitalized $\mathrm{HeFH}$ patients with COVID-19, particularly those with an elevated Lp(a) level and a history of an ACS.

\section{ANTIPLATELET DRUGS}

A recent meta-analysis was carried out to find the effect of antiplatelet treatments on patients with COVID-19 infection (41). In this analysis, the treatments included acetylsalicylic acid and P2Y12 inhibitors. The meta-analysis included nine articles and 5,970 patients, and it showed that the use of the studied antiplatelet drugs did not associate with a reduced risk for the severe form of COVID-19 (OR = 0.98, 95\% CI: 0.64-1.50, $p=$ $\left.0.94 ; I^{2}=65 \%\right)$. This result resembles that obtained in a Japanese cohort study of a total of 4,265 hospitalized patients (42). In this latter analysis, the use of antiplatelet drugs failed to impact the severity of COVID-19.

Anticoagulants have been more potent in inhibiting both venous thrombosis and fibrin formation in critical vessels, while antiplatelet agents alone seem unable to control the viral procoagulant action. Statins, with their multiple calmingdown influences, which occur via their anti-inflammatory and especially via their tissue factor synthesis-reducing actions, provide a multitargeted adjunct therapeutic opportunity (28). However, any clinical meta-analysis faces the problem of pooling various risk category patients together, which, in the case of trying to balance the bleeding and thrombosis risks, may neutralize the personalized needs and benefits of a medication. The highest risk patient would likely benefit most from a combination therapy involving both an antiplatelet drug and a statin, in addition to thromboprophylactic doses of low-molecular-weight heparin (43). Some cardiovascular patients, and even those with subclinical atherosclerosis, also have atrial fibrillation, which often requires anticoagulation (44). Accordingly, statins need to be used in association with anticoagulation, at least in these high-risk hypercholesterolemic patients. In coagulation, the mechanisms of thrombin formation are synergistic rather than additive. Therefore, the inhibition of coagulation calls upon combination strategies to alleviate thrombotic complications in the hospitalized high-risk $\mathrm{HeFH}$ patients with COVID19. The further role of low-molecular-weight heparin, at the latest during hospital admission, would allow protection against cardiovascular complications $(45,46)$.

\section{CONCLUDING REMARKS}

Given the increased baseline incidence of VTE in $\mathrm{HeFH}$ due to the presence of a lifelong severe hypercholesterolemia, the benefits gained by efficient cholesterol-lowering therapy in the $\mathrm{HeFH}$ patients are likely to exceed that of the patients with a diet-induced hypercholesterolemia, in whom statin use is also advocated. Indeed, observational populationbased cohort studies show that statin treatment decreases recurrent VTE, which carries an especially high reoccurrence rate of around $15-20 \%(47,48)$. Another interesting piece of information on the favorable effect of cholesterol-lowering therapy comes from the recent post-hoc analysis of the FOURIER and ODYSSEUS OUTCOMES (49). In this analysis, a $31 \%$ relative risk reduction of VTE with PCSK9 inhibition [HR, 0.69 (95\% CI, 0.53-0.90); $p=0.017]$ was observed. The risk reduction particularly applied to carriers of high serum $\mathrm{Lp}$ (a) who benefitted from the PCSK9 treatment to prevent recurrent VTEs.

Overall, an increased risk of bleeding complications occurs in about $5-8 \%$ of the COVID-19 patients (50). Thus, statin and PCSK9 inhibitor treatment for the lowering of LDL-C levels, when used together with a strong antithrombotic medication, may impair hemostasis and cause intracerebral bleeds which are mostly fatal in COVID-19 (51, 52). Based on the above considerations, the use of these medications should be personalized to safely master the critical and delicate balance between the unphysiological coagulation of thrombosis and bleeding tendency in an individual COVID-19 patient. This analysis is based on the medical history of the patient and his family, potentially untreated hypertension, actual lipid levels, hepatic (prolonged prothrombin time) and renal impairment, and an analysis of relevant coagulation laboratory tests and exclusion of anemia and thrombocytopenia, which together with low fibrinogen and von Willebrand factor (usually high in COVID-19) levels refer to a bleeding risk.

Several reasons support the use of efficient cholesterollowering treatment in $\mathrm{HeFH}$ patients with COVID-19. The prehospitalization use of statins has recently been observed to reduce mortality in COVID-19 by more than $50 \%$ (20). We wish to highlight the extra benefit of statin use in the prevention of potential venous thrombotic events. So far, continued cholesterol lowering in hospitalized HeFH with COVID-19 has been suggested. It seems to be justified considering the enhancement of cholesterol-lowering medication in these patients also after discharge from the hospital, as COVID-19 may cause a longterm increased cardiovascular risk on top of the preexisting high cardiovascular risk in $\mathrm{HeFH}$ patients $(19,53,54)$. While the specific information on the role of cholesterol-lowering therapy in the VTE risk is being gathered, effective means to lower plasma cholesterol levels are likely to be paramount for improved prognosis among HeFH patients with COVID-19.

Finally, combining cholesterol-lowering and antithrombotic medications when attempting to prevent the often-lethal thrombotic complications of COVID-19 is prudent. This particularly applies to statins and, on a discretional basis, also to the PCSK9 inhibitors, which possess a combined LDL-Cand $\mathrm{Lp}(\mathrm{a})$-lowering effect, thereby gaining potentially unique antithrombotic effects that inhibit both coagulation and platelet activation $(28,55)$. In this way, both statins and PCSK9 inhibitors are valuable adjuncts to the overall antithrombotic armamentarium in hospitalized hypercholesterolemic COVID19 patients, particularly in COVID-19 patients with severe hypercholesterolemia-whether or not they have the diagnosis of HeFH. In these patients, for short-term and long-term prophylaxis against thrombosis, the treating physicians need to bear in mind not only the traditional preventive target 
vessels, i.e., those in the arterial system, but also those in the venous system, and without forgetting the risks of bleeding complications. Thus, any individual treatment decision needs to be made under the vigilance of the delicate hemostatic balance.

\section{REFERENCES}

1. Brown MS, Kovanen PT, Goldstein JL. Regulation of plasma cholesterol by lipoprotein receptors. Science. (1981) 212:62835. doi: 10.1126/science.6261329

2. Vuorio AF, Turtola H, Kontula K. Neonatal diagnosis of familial hypercholesterolemia in newborns born to a parent with a molecularly defined heterozygous familial hypercholesterolemia. Arterioscler Thromb Vasc Biol. (1997) 17:3332-7. doi: 10.1161/01.ATV.17.11.3332

3. Hu P, Dharmayat KI, Stevens CAT, Sharabiani MT, Jones RS, Watts GF, et al. Prevalence of familial hypercholesterolemia Among the general population and patients with atherosclerotic cardiovascular disease: a systematic review and meta-analysis. Circulation. (2020) 141:1742-59. doi: 10.1161/CIRCULATIONAHA.119.044795

4. Representatives of the Global Familial Hypercholesterolemia Community, Wilemon KA, Patel J, Aguilar-Salinas C, Ahmed CD, Alkhnifsawi M, et al. Reducing the clinical and public health burden of familial hypercholesterolemia: a global call to action. JAMA Cardiol. (2020) 5:21729. doi: 10.1001/jamacardio.2019.5173

5. Tada H, Kawashiri MA, Okada H, Teramoto R, Konno T, Yoshimuta $\mathrm{T}$, et al. Assessment of coronary atherosclerosis in patients with familial hypercholesterolemia by coronary computed tomography angiography. Am J Cardiol. (2015) 115:724-9. doi: 10.1016/j.amjcard.2014.12.034

6. Vuorio A, Watts GF, Schneider WJ, Tsimikas S, Kovanen PT. Familial hypercholesterolemia and elevated lipoprotein(a): double heritable risk and new therapeutic opportunities. J Intern Med. (2020) 287:2-18. doi: 10.1111/joim.12981

7. Boffa MB, Koschinsky ML. Oxidized phospholipids as a unifying theory for lipoprotein(a) and cardiovascular disease. Nat Rev Cardiol. (2019) 16:30518. doi: 10.1038/s41569-018-0153-2

8. Etingin OR, Hajjar DP, Hajjar KA, Harpel PC. Nachman RL. Lipoprotein (a) regulates plasminogen activator inhibitor-1 expression in endothelial cells A potential mechanism in thrombogenesis. J Biol Chem. (1991) 266:245965. doi: 10.1016/S0021-9258(18)52266-3

9. Rosenson RS, Goonewardena SN. The residual risk Odyssey: from LDL to Lp(a). J Am Coll Cardiol. (2021) 78:434-6. doi: 10.1016/j.jacc.2021.04.103

10. Humphries SE, Cooper JA, Seed M, Capps N, Durrington PN, Jones B, et al. Coronary heart disease mortality in treated familial hypercholesterolaemia: update of the UK Simon Broome FH register. Atherosclerosis. (2018) 274:416. doi: 10.1016/j.atherosclerosis.2018.04.040

11. Vuorio A, Kuoppala J, Kovanen PT, Humphries SE, Tonstad S, Wiegman A, et al. Statins for children with familial hypercholesterolemia. Cochrane Database Syst Rev. (2019) 11:CD006401. doi: 10.1002/14651858.CD006401.pub5

12. Vuorio A, Watts GF, Kovanen PT. Initiation of PCSK9 inhibition in patients with heterozygous familial hypercholesterolaemia entering adulthood: a new design for living with a high-risk condition? Eur Heart J. (2016) 37:13536. doi: 10.1093/eurheartj/ehw010

13. Luirink IK, Wiegman A, Kusters DM, Hof MH, Groothoff JW, de Groot E, et al. 20-year follow-up of statins in children with familial hypercholesterolemia. N Engl J Med. (2019) 381:1547-56. doi: 10.1056/NEJMoa1816454

14. Santos RD, Stein EA, Hovingh GK, Blom DJ, Soran H, Watts GF, et al. Longterm evolocumab in patients with familial hypercholesterolemia. J Am Coll Cardiol. (2020) 75:565-74. doi: 10.1016/j.jacc.2019.12.020

15. Kunutsor SK, Laukkanen JA. Incidence of venous and arterial thromboembolic complications in COVID-19: A systematic review and metaanalysis. Thromb Res. (2020) 196:27-30. doi: 10.1016/j.thromres.2020.08.022

16. Piroth L, Cottenet J, Mariet AS, Bonniaud P, Blot M, TubertBitter $\mathrm{P}$, et al. Comparison of the characteristics, morbidity, and mortality of COVID-19 and seasonal influenza: a nationwide,

\section{AUTHOR CONTRIBUTIONS}

AV and PK: writing the first draft. AV, RL, and PK: editing to produce the final draft. All authors contributed to the article and approved the submitted version.

population-based retrospective cohort study. Lancet Respir Med. (2021) 9:251-9. doi: 10.1016/S2213-2600(20)30527-0

17. Prandoni P. Venous and arterial thrombosis: two aspects of the same disease? Clin Epidemiol. (2009) 1:1-6. doi: 10.2147/CLEP.S4780

18. Chen S, Zhang D, Zheng T, Yu Y, Jiang J. DVT incidence and risk factors in critically ill patients with COVID-19. J Thromb Thrombolysis. (2021) 51:33-9. doi: 10.1007/s11239-020-02181-w

19. Tan BK, Mainbourg S, Friggeri A, Bertoletti L, Douplat M, Dargaud Y, et al. Arterial and venous thromboembolism in COVID-19: a study-level meta-analysis. Thorax. (2021) 76:970-9. doi: 10.1136/thoraxjnl-2020-215383

20. Gupta A, Madhavan MV, Poterucha TJ, DeFilippis EM, Hennessey JA Redfors B, et al. Association between antecedent statin use and decreased mortality in hospitalized patients with COVID-19. Res Sq. (2021) 11:rs3.rs56210. doi: 10.1038/s41467-021-21553-1

21. Iqbal Z, Ho JH, Adam S, France M, Syed A, Neely D, et al. Managing hyperlipidaemia in patients with COVID-19 and during its pandemic: An expert panel position statement from HEART UK. Atherosclerosis. (2020) 313:126-36. doi: 10.1016/j.atherosclerosis.2020.09.008

22. Kow CS, Hasan SS. Meta-analysis of effect of statins in patients with COVID19. Am J Cardiol. (2020) 134:153-5. doi: 10.1016/j.amjcard.2020.08.004

23. Masana L, Correig E, Rodríguez-Borjabad C, Anoro E, Arroyo JA, Jericó C, et al. Effect of statin therapy on SARS-CoV-2 infection-related mortality in hospitalized patients. Eur Heart J Cardiovasc Pharmacother. (2020). doi: 10.1093/ehjcvp/pvaa128. [Epub ahead of print].

24. Vuorio A, Kovanen PT. Statins as adjuvant therapy for COVID-19 to calm the stormy immunothrombosis and beyond. Front Pharmacol. (2021) 11:579548. doi: 10.3389/fphar.2020.579548

25. Sorensen KE, Celermajer DS, Georgakopoulos D, Hatcher G, Betteridge DJ, Deanfield JE. Impairment of endothelium-dependent dilation is an early event in children with familial hypercholesterolemia and is related to the lipoprotein(a) level. J Clin Invest. (1994) 93:50-5. doi: 10.1172/JCI1 16983

26. Ray JG, Mamdani M, Tsuyuki RT, Anderson DR, Yeo EL, Laupacis A. Use of statins and the subsequent development of deep vein thrombosis. Arch Intern Med. (2001) 161:1405-10. doi: 10.1001/archinte.161.11.1405

27. Undas A, Brummel-Ziedins KE, Mann KG. Statins and blood coagulation. Arterioscler Thromb Vasc Biol. (2005) 25:28794. doi: 10.1161/01.ATV.0000151647.14923.ec

28. Owens AP 3rd, Passam FH, Antoniak S, Marshall SM, McDaniel AL, Rudel $\mathrm{L}$, et al. Monocyte tissue factor-dependent activation of coagulation in hypercholesterolemic mice and monkeys is inhibited by simvastatin. J Clin Invest. (2012) 122:558-68. doi: 10.1172/JCI58969

29. Ferrari F, Martins VM, Teixeira M, Santos RD, Stein R. COVID-19 and thromboinflammation: Is there a role for statins? Clinics. (2021) 76:e2518. doi: 10.6061/clinics/2021/e2518

30. Loppnow $H$, Zhang L, Buerke $M$, Lautenschläger $M$, Chen L, Frister A, et al. Statins potently reduce the cytokine-mediated IL-6 release in SMC/MNC cocultures. J Cell Mol Med. (2011) 15:994-1004. doi: 10.1111/j.1582-4934.2010.01036.x

31. Mi Y, Yan S, Lu Y, Liang Y, Li C. Venous thromboembolism has the same risk factors as atherosclerosis: a PRISMA-compliant systemic review and metaanalysis. Medicine. (2016) 95:e4495. doi: 10.1097/MD.0000000000004495

32. Vuorio A, Raal F, Kaste M, Kovanen PT. Familial hypercholesterolaemia and COVID-19: a two-hit scenario for endothelial dysfunction amenable to treatment. Atherosclerosis. (2021) 320:5360. doi: 10.1016/j.atherosclerosis.2021.01.021

33. Nordestgaard BG, Chapman MJ, Humphries SE, Ginsberg HN, Masana L, Descamps OS, et al. Familial hypercholesterolaemia is underdiagnosed and undertreated in the general population: guidance for clinicians to prevent 
coronary heart disease: consensus statement of the European Atherosclerosis Society. Eur Heart J. (2013) 34:3478-90a. doi: 10.1093/eurheartj/eht273

34. Kawasaki T, Kambayashi J, Ariyoshi H, Sakon M, Suehisa E, Monden M. Hypercholesterolemia as a risk factor for deep-vein thrombosis. Thromb Res. (1997) 88:67-73. doi: 10.1016/S0049-3848(97)00192-8

35. Lassila R, Jula A, Pitkäniemi J, Haukka J. Statin use associates with reduced incidence of venous thromboembolism - a nationwide population-based cohort study. BMJ Open. (2014) 4:e005862. doi: 10.1136/bmjopen-2014-005862

36. Krogh HW, Mundal L, Holven KB, Retterstøl K. Patients with familial hypercholesterolaemia are characterized by presence of cardiovascular disease at the time of death. Eur Heart J. (2016) 37:1398-405. doi: 10.1093/eurheartj/ehv602

37. Vuorio A, Kovanen PT. Prevention of endothelial dysfunction and thrombotic events in COVID-19 patients with familial hypercholesterolemia. J Clin Lipidol. (2020) 14:617-8. doi: 10.1016/j.jacl.2020.06.006

38. Raal FJ, Stein EA, Dufour R, Turner T, Civeira F, Burgess L, et al. PCSK9 inhibition with evolocumab (AMG 145) in heterozygous familial hypercholesterolaemia (RUTHERFORD-2): a randomised, double-blind, placebo-controlled trial. Lancet. (2015) 385:331-40. doi: 10.1016/S0140-6736(14)61399-4

39. Schol-Gelok S, van der Hulle T, Biedermann JS, van Gelder T, Klok FA, van der Pol LM, et al. Clinical effects of antiplatelet drugs and statins on D-dimer levels. Eur J Clin Invest. (2018) 48:e12944. doi: 10.1111/eci.12944

40. Schwartz GG, Szarek M, Bittner VA, Diaz R, Goodman SG, Jukema JW, et al. Lipoprotein(a) and benefit of PCSK9 inhibition in patients with nominally controlled LDL cholesterol. J Am Coll Cardiol. (2021) 78:42133. doi: 10.1016/j.jacc.2021.04.102

41. Wang Y, Ao G, Nasr B, Qi X. Effect of antiplatelet treatments on patients with COVID-19 infection: a systematic review and meta-analysis. Am J Emerg Med. (2021) 43:27-30. doi: 10.1016/j.ajem.2021.01.016

42. Togano T, Uemura Y, Asai Y, Hayakawa K, Matsunaga N, Terada M, et al. The influence of pre-admission antiplatelet and anticoagulation therapy on the illness severity in hospitalized patients with COVID-19 in Japan. J Infect Chemother. (2021) 27:1498-503. doi: 10.1016/j.jiac.2021.07.016

43. Chistolini A, Ruberto F, Alessandri F, Santoro C, Barone F, Puzzolo C, et al. Effect of low or high doses of low-molecular-weight heparin on thrombin generation and other haemostasis parameters in critically ill patients with COVID-19. Br J Haematol. (2020) 190:e214-8. doi: 10.1111/bjh.17003

44. Kristensen KE, Knage CC, Nyhegn LH, Mulder BA, Rienstra M, Van Gelder IC, et al. Subclinical atherosclerosis is associated with incident atrial fibrillation: a systematic review and meta-analysis. Europace. (2020) 22:9911000. doi: 10.1093/europace/euaa030

45. ATTACC Investigators, ACTIV-4a Investigators, REMAP-CAP Investigators. Therapeutic anticoagulation with heparin in noncritically Ill patients with Covid-19. N Engl J Med. (2021) 385:790-802. doi: 10.1056/NEJMoa2105911

46. Vuorio A, Raal F, Kovanen PT. Hospitalized children with familial hypercholesterolemia and COVID-19: a case for preventive anticoagulation. Front Cardiovasc Med. (2021) 8:657719. doi: 10.3389/fcvm.2021.657719

47. Biere-Rafi S, Hutten BA, Squizzato A, Ageno W, Souverein PC, de Boer A, et al. Statin treatment and the risk of recurrent pulmonary embolism. Eur Heart J. (2013) 34:1800-6. doi: 10.1093/eurheartj/eht046
48. Nguyen CD, Andersson C, Jensen TB, Gjesing A, Schjerning Olsen AM, Malta Hansen C, et al. Statin treatment and risk of recurrent venous thromboembolism: a nationwide cohort study. BMJ Open. (2013) 3:e003135. doi: 10.1136/bmjopen-2013-003135

49. Marston NA, Gurmu Y, Melloni GEM, Bonaca M, Gencer B, Sever PS, et al. The effect of PCSK9 (Proprotein Convertase Subtilisin/Kexin Type 9) inhibition on the risk of venous thromboembolism. Circulation. (2020) 141:1600-7. doi: 10.1161/CIRCULATIONAHA.120.046397

50. Al-Samkari H, Karp Leaf RS, Dzik WH, Carlson JC, Fogerty AE, Waheed A, et al. COVID-19 and coagulation: bleeding and thrombotic manifestations of SARS-CoV-2 infection. Blood. (2020) 136:489-500. doi: 10.1182/blood.2020006520

51. Ma C, Gurol ME, Huang Z, Lichtenstein AH, Wang X, Wang $\mathrm{Y}$, et al. Low-density lipoprotein cholesterol and risk of intracerebral hemorrhage: a prospective study. Neurology. (2019) 93:e445-57. doi: 10.1212/WNL.0000000000007853

52. Leasure AC, Khan YM, Iyer R, Elkind MS, Sansing LH, Falcone GJ, et al. Intracerebral hemorrhage in patients with COVID-19: an analysis from the COVID-19 cardiovascular disease registry. Stroke. (2021) 52:e3213. doi: 10.1161/STROKEAHA.121.034215

53. Vuorio A, Watts GF, Kovanen PT. Familial hypercholesterolaemia and COVID-19: triggering of increased sustained cardiovascular risk. J Intern Med. (2020) 287:746-7. doi: 10.1111/joim.13070

54. Banach M, Penson PE, Fras Z, Vrablik M, Pella D, Reiner Ž, et al. Brief recommendations on the management of adult patients with familial hypercholesterolemia during the COVID-19 pandemic. Pharmacol Res. (2020) 158:10489. doi: 10.1016/j.phrs.2020.104891

55. Violi F, Calvieri C, Ferro D, Pignatelli P. Statins as antithrombotic drugs. Circulation. (2013) 127:251-7. doi: 10.1161/CIRCULATIONAHA.112.145334

Conflict of Interest: PK has received lecture honoraria and/or travel fees from Amgen, Novartis, Raisio Group, and Sanofi. RL has received lecture honoraria and served in advisory boards of Alexion, Astra Zeneca, Bayer, Pfizer, Roche, and Sanofi.

The remaining author declares that the research was conducted in the absence of any commercial or financial relationships that could be construed as a potential conflict of interest.

Publisher's Note: All claims expressed in this article are solely those of the authors and do not necessarily represent those of their affiliated organizations, or those of the publisher, the editors and the reviewers. Any product that may be evaluated in this article, or claim that may be made by its manufacturer, is not guaranteed or endorsed by the publisher.

Copyright (c) 2021 Vuorio, Lassila and Kovanen. This is an open-access article distributed under the terms of the Creative Commons Attribution License (CC BY). The use, distribution or reproduction in other forums is permitted, provided the original author(s) and the copyright owner(s) are credited and that the original publication in this journal is cited, in accordance with accepted academic practice. No use, distribution or reproduction is permitted which does not comply with these terms. 